\title{
El pensamiento cartesiano y la fundación de la sociología
}

\section{Cartesian thought and the foundation of sociology}

Loreto Arias-Lagos (loreto.arias@gmail.com) Facultad de Educación, Ciencias Sociales y Humanidades, Universidad de La Frontera (Temuco, Chile) https://orcid.org/0000-0003-4999-0941

Felipe Sáez-Ardura (inti.felipe@gmail.com) Facultad de Ciencias Sociales y Humanidades, Universidad Autónoma de Chile (Temuco, Chile) https://orcid.org/0000-0001-9472-5552

Juan Peña-Axt (juan.pena@uautonoma.cl) Facultad de Ciencias Sociales y Humanidades, Universidad Autónoma de Chile (Temuco, Chile) https://orcid.org/0000-0002-7689-566X

\begin{abstract}
This paper analyses the foundations of the so-called mathesis universalis, and how this notion, proposed by Descartes in the 17th century, has had significant implications in the origin and constitution (much later) of two of the most important versions of sociology: sociological positivism and comprehensive sociology. Maintaining that the discipline that studies social phenomena has been constantly influenced by Cartesian thought, some postulates of both approaches are analysed based on the indicated principle. It is concluded that, despite being diametrically opposed from the point of view of the ontological principles on which they observe social reality, in both lines of sociological analysis the principle of mathesis universalis remains in force. This shows an evident logic of critical continuity of Cartesianism in the foundational approaches of sociology, all of which has turned out to be epistemologically significant for the discipline that aims to scientifically study social facts.
\end{abstract}

Key words: Descartes, clear ideas, distinct, ideas, universal mathesis, sociology.

\section{Resumen}

Resulta de interés para el presente trabajo analizar los fundamentos de la denominada mathesis universalis y cómo esta noción, propuesta por Descartes en pleno siglo XVII, ha tenido implicancias significativas en el origen y constitución (muy ulterior) de dos de las versiones más importantes de la sociología: el positivismo sociológico y la sociología comprensiva. Sosteniendo que la disciplina que estudia los fenómenos sociales ha estado influenciada constantemente por el pensamiento cartesiano, se analizan algunos postulados de ambos enfoques con base en el principio indicado. Se concluye que, pese a ser diametralmente opuestas desde el punto de vista de los principios ontológicos sobre los que observan la realidad social, en ambas líneas de análisis sociológico el principio de la mathesis universalis sigue vigente. Esto da cuenta de una evidente lógica de continuidad crítica del cartesianismo en los enfoques fundacionales de la sociología, todo lo cual ha resultado ser epistemológicamente significativo para la disciplina que se encamina a estudiar científicamente los hechos sociales.

Palabras clave: Descartes, ideas claras, ideas distintas, mathesis universalis, sociología. 


\section{Introducción}

A partir de los libros denominados Reglas para la dirección del espíritu y Meditaciones metafísicas, escritas por Descartes durante el prolífico siglo XVII europeo, se iniciará en la filosofía una nueva concepción de la realidad y de la forma de acceder a ella. Estos trabajos permitirán, en un contexto de consolidación del método científico, una apertura matematizante a la llamada res extensa, lo cual no es menor en la historia del pensamiento moderno. Dicha apertura radica, básicamente, en el postulado de la Mathesis Universalis, afirmación que termina siendo un eje central en el desarrollo de la filosofía cartesiana.

El presente trabajo aborda las consideraciones sociológicas y epistemológicas centrales que relevan este postulado cartesiano, el cual termina por perfilar no solo la naturaleza misma de la ciencia contemporánea, sino que también, según se plantea en este escrito, muy particularmente el proceso mismo de instauración del pensamiento sociológico fundacional, acaecido, en tanto pleno apogeo de dicho pensamiento, durante la segunda mitad del siglo XIX y las primeras décadas del XX. Es en ese momento en el que aparecieron obras clásicas elementales de la sociología, cuya visión de la sociedad moderna queda retratada en trabajos de trascendencia histórica en la disciplina, tales como el Discours sur l'esprit positif, publicado en el año 1844 por A. Comte o Les règles de la méthode sociologique, publicado más de 40 años después por É. Durkheim.

Lo que queda demostrado en obras como esas, finalmente, refiere a que la constitución disciplinaria misma genera un esfuerzo para quedar 'divorciada' de una filosofía social e histórica marcada fuertemente por el positivismo. Dicho esfuerzo termina consolidándose, en pos del nacimiento de una 'ciencia independiente', sobre la base de la definición teórica y metodológica indudablemente matematizante de la realidad social moderna, por lo menos para lo que refiere al caso específico de la sociología en tres de sus teorías clásicas esenciales: el sistema comteano, el sistema spenceriano y el sistema durkheimiano, sin mencionar el enfoque weberiano que, con Schütz principalmente, aboga por un superación del sistema cartesiano sobre la base de la comprensión, yendo por un camino diferente al positivismo sociológico. Dado ello, es legítimo hacerse la siguiente pregunta: ¿qué elementos se conjugan en la Mathesis Universalis, en tanto forma cartesiana de acceso a la res extensa, y cómo se implica este relevante posicionamiento filosófico con el origen de la sociología fundacional?

Esta cuestión requiere ser revisada, ya que es pertinente para comprender de forma más acabada el pensamiento cartesiano en el origen y consolidación de la ciencia sociológica. Los argumentos que a continuación se exponen buscan alimentar esta discusión, abriendo un camino a la reflexión en torno a la relevancia que sigue teniendo el pensamiento de Descartes para las ciencias sociales en general, y para la sociología científica en particular. Para esto, y en función de la pregunta planteada, el escrito desarrolla tres argumentos centrales: (1) para entender la Mathesis, se debe dar cuenta de la concepción matematizante de la realidad en función de las llamadas ideas claras y distintas. (2) La Mathesis Universalis, en el proyecto cartesiano, refiere a la finalidad metódica de acceder a la verdad de la res extensa. (3) Ambos elementos condicionan la pretensión de validez de la sociología, en tanto ciencia de los hechos sociales, por lo que el sistema cartesiano vive en el origen de esta disciplina un intenso proceso de continuidad y ruptura epistemológica, materializada en la emergencia de una sociología positiva y otra interpretativa. Este análisis se realizará a partir de la Meditación Segunda "De la naturaleza del espíritu; y que es más fácil de conocer que el cuerpo", la Meditación Tercera "De Dios, que existe", la Meditación Cuarta "De lo verdadero y de lo 
falso" y la Meditación Quinta "De la esencia de las cosas materiales; y otra vez de la existencia de Dios". También se han trabajado las cuatro primeras reglas que enuncia Descartes en Reglas para la dirección del espíritu.

\section{Sobre las ideas claras y distintas}

En las Meditaciones Tercera y Cuarta, Descartes plantea la inquietud respecto a la distinción de las ideas y de las cosas que están en el pensamiento, pudiendo ser éstas distintas o confusas, claras u oscuras. En términos metafísicos, el despliegue epistemológico de esta tesis permite entender el papel de la distinción en el proyecto cartesiano, encarnado en la Mathesis Universalis. Descartes, al respecto, señalará: "por ello me parece poder establecer desde ahora, como regla general, que son verdaderas todas las cosas que concebimos muy clara y distintivamente" (Descartes 1977:31).

Así, esta distinción no solo es teóricamente útil, sino que además da cuenta de la prioridad existente entre las ciencias. Aquellas que tratan de cosas simples y generales, tienen un privilegio epistemológico por sobre las ciencias que dan cuenta de cosas compuestas, "dudosas e inciertas" (Descartes 1977:19), lo cual permite afirmar un paso necesario para el despliegue de la ciencia moderna, al dar cuenta de criterios básicos para distinguir conocimientos verdaderos de conocimientos falsos, los cuales, como es sabido, constituyen la pretensión de todas las ciencias en cuanto ciencias. No por nada Hegel, en su Introduction to the philosophy's history, publicada casi 150 años después de las obras de Descartes, seguía afirmando muy convencido que la filosofía comienza "solamente allí donde la esencia de las cosas llega a la conciencia en la forma de pensamiento puro" (Hegel 1984:151). Aquí se aprecia un componente sustantivo de la constitución del principio de 'objetividad' en la sociología posterior, recalcadas, por ejemplo, por Durkheim cuando afirmaba que una empresa semejante (la de establecer principios teorético-metodológicos para una 'ciencia independiente'), "puede y debe ser acogida sin inquietud, y hasta con simpatía, por todos los que, pese a que se aparten de nosotros en algunos puntos, comparten nuestra fe en el futuro de la razón" (Durkheim 2001:11).

Este primer arranque, en sí mismo, trata del despliegue del conocimiento respecto del mundo, y lo entrega Descartes al dar cuenta de las ideas claras y distintas como requisito para la verdad, ideas que no sucumben ante la posibilidad de la duda, definiendo que "es solo la razón, no los sentidos, lo que permite establecer el ser de las cosas, o como lo pone Descartes, las ideas claras y distintas de las mismas" (González 2016:170). El quiebre epistemológico instituido por Descartes, particularmente en las Meditaciones Primera y Segunda, lleva a dudar de lo entendido como cierto hasta ese minuto. Esto incluye al espíritu mismo y a todo lo que se concibe como parte de la sólida base de las ciencias con pretensiones de verdad. Cualquier conocimiento que se llame científico debe hacer frente a la posibilidad de la duda, ya que el acto de dudar, vale decir, el ejercicio de dudar metódicamente, se considera un ejercicio con pretensiones de cientificidad, y se plantea consecuentemente como un requerimiento para la ciencia contemporánea. Es decir, dado que el despliegue científico encuentra uno de sus impulsos para su concreción, dudar de lo ya establecido se considera certero para el ejercicio del saber.

De esta manera, afirma Descartes, "duerma yo o esté despierto, dos más tres serán siempre cinco, y el cuadrado no tendrá más de cuatro lados; no pareciendo posible que verdades tan patentes puedan ser sospechosas de falsedad o incertidumbre alguna" (Descartes 1977:19). Esta afirmación lleva a pensar en la posibilidad cierta de que los sentidos nos engañen, lo cual se traduce a establecer 
aprehensiones respecto del mundo que son inciertas, como un estado de ensueño. A la vez, se plantea la existencia de verdades tan irrefutables que no puedan ponerse en duda. Entonces, si bien a través de los sentidos podemos tocar, oír y explorar un cuerpo o un objeto, conociendo sus características sensibles, este acto no nos permite acceder a la verdadera naturaleza del objeto. La naturaleza cambiante de lo que percibimos, a partir de nuestras capacidades sensoriales, parece ser una muestra o una expresión de esta desconfianza en los sentidos, lo que en palabras de Descartes termina siendo categórico: "es prudente no fiarse nunca de quienes nos han engañado una vez" (Descartes 1977:18).

Dado lo anterior, estas posibilidades de acercamiento al mundo -lo falso y lo verdadero, en el sistema conceptual de Descartes- llevan necesariamente a la pregunta sobre la distinción de las ideas claras y las distintas en la tarea cognoscente. Esta distinción Descartes la ejemplifica y proyecta en la figura de un triángulo. Es posible imaginarlo, sin incluso verlo, y no por ello la figura deja de tener cierta naturaleza o esencia, la cual es inmutable y eterna. El triángulo en sí no depende del espíritu y eso es evidente porque pueden demostrarse dichas propiedades de ese triángulo, "aunque con anterioridad no se haya pensado en ellas" (Descartes 1977:55). De este modo, no es posible separar el triángulo de sus propiedades matemáticas. La claridad de las propiedades matemáticas del triángulo lleva, según el autor, a reconocer de forma evidente y clara las propiedades que están en él. Entonces, el acceso a la verdad está dado por las cosas que se presentan con claridad y distinción, lo cual no permite errar en la consideración de múltiples interpretaciones. En Descartes, entonces, la claridad y la distinción entregan las claves para un acercamiento epistémico verdadero al mundo, y el logro de esa certeza es otorgada por la explicación matematizante sobre esta res extensa.

Si bien el autor posiciona una forma de acercamiento al mundo, a partir de las ideas claras y distintas, pareciera ser que necesita de certezas respecto a la existencia de Dios, de modo de dar una base que no permite ningún ápice de incertidumbre. En Meditaciones metafísicas, Descartes alude a la idea de Dios, asunto apenas mencionado en las Reglas para la dirección del espíritu. Al igual que las matemáticas, la existencia de Dios es algo cierto; no es posible separar la existencia de su esencia, así como no es posible separar el triángulo de sus propiedades matemáticas. Por lo tanto, como no es posible entender a Dios sin la existencia, Dios existe y es perfección porque existe. Entonces, todo lo que viene de Dios es necesariamente verdadero, comprendido de forma clara y distintivamente, por lo que el conocimiento de Dios abre la posibilidad de adquirir "una ciencia perfecta acerca de infinidad de cosas" (Descartes 1977:59).

Esta afirmación es relevante, ya que da cuenta de la forma en la que el Descartes de las Meditaciones metafísicas encuentra el piso necesario en la idea de la existencia de Dios, como función epistemológica dirigida a amparar la relación entre el espíritu y las cosas del mundo. Al respecto, señala lo siguiente: "ya descubro un camino que me conducirá desde esa contemplación de Dios verdadero (en quien están encerrados todos los tesoros de la ciencia y la sabiduría) al conocimiento de las restantes cosas del mundo" (Descartes 1977:45). Por lo tanto, Dios es garante para el conocimiento de la res extensa.

Esta responsabilidad atribuida al cogito lleva a preguntarse, en el marco del sistema cartesiano, por el cómo se garantiza el correcto ejercicio de la conciencia para distinguir lo claro de lo distinto. Descartes, en este plano de la reflexión, recurre a la idea de Dios, y parece ser garante de la capacidad del cogito para distinguir y encauzar el espíritu hacia cosas claras y distintas. Bajo esta 


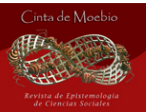

concepción del conocimiento, la idea de Dios y su perfección deviene del cogito. Ahora bien, el criterio de claridad y distinción, antes expuesto, lleva a la interrogante respecto de quién está detrás de esta distinción. La respuesta no es otra que la cosa pensante, la res cogitans, por lo que se entiende que sea el cogito quien, a partir de la certeza, como cosa pensante, demuestre no solo su existencia, sino que también la posibilidad de distinguir lo verdadero de lo falso, en tanto conciencia. Vale decir, es en su espíritu donde la cosa pensante debe buscar para dar cuenta de la verdad, que, al ser clara y distinta, es evidente.

Es posible aseverar en esta discusión una reafirmación del cogito frente a la tarea cognoscente, siendo el método propuesto por Descartes el que abre un camino para la objetividad. El ejercicio de la duda metódica en este ámbito, y la superación de las incertidumbres en el ejercicio cognoscente, entregan la posibilidad para deshacernos de prejuicios y percepciones erróneas frente al mundo, algo que es muy propio en el sentido común. De este modo, es el cogito el encargado de encauzar el método. Descartes no abandona el solipsismo que caracteriza su pensamiento, indicando que "basta con poder distinguir claramente una cosa de otra, para estar seguro que la una es diferente de la otra" (Descartes 1977:65). La conciencia, de este modo, tendría la capacidad de distinguir con claridad.

\section{La certeza de la Mathesis Universalis como acceso a la verdad}

Teniendo como base las categorías anteriores referidas a las ideas claras y distintas (suelo firme desde la cual erige el pensador sus postulados sobre el conocimiento verdadero), es preciso aclarar una distinción relevante, y es la que realiza Descartes en la Regla IV entre matemáticas corrientes y la Mathesis, la cual permitirá comprender el papel de esta última en el proyecto cartesiano, y con ello el alcance de su propuesta para la sociología fundacional. La Mathesis Universalis se refiere a "cierta ciencia general que explique todo lo que puede buscarse acerca del orden y la medida no adscrito a la materia especial" (Descartes 1997:86). Aquí aparece algo tremendamente problemático, que tiene que ver con que, para Descartes, pareciera quedar en clara evidencia un hecho fundamental para llegar a la verdad de la res extensa, a saber, que no sería necesario volcarse a la experiencia del mundo para acceder a la verdad de las cosas. Vale decir, no es requerimiento ir a la materia para poder acceder al mundo, lo que en una primera impresión podría entrar en una fuerte contradicción con el acceso objetivo a la verdad de las cosas en la conciencia, tan aceptada por la sociología fundacional en su etapa de consolidación disciplinaria, la cual, según Comte, debía dirigirse hacia el establecimiento de "un régimen definitivo de la razón humana" (Comte 2013a:33).

La condición de esta Mathesis es la de constituirse como una ciencia general, dando indicios de la unicidad del método en la propuesta cartesiana, especialmente según lo que desarrolla el pensador en la Regla IV, que es donde afirma la existencia de una vía al conocimiento certero. La noción de orden, de igual forma, hace referencia a la existencia de un método. Por tanto, la Mathesis no es solo números o figuras que atañen más a la imaginación que al entendimiento (haciendo alusión directa a la matemática corriente), sino que más bien representa "ciertas semillas de verdades impresas por la naturaleza en el espíritu humano" (Descartes 1977:84). Esto remite a la presencia, en la mentada Mathesis, de un innatismo cartesiano, afirmando la posibilidad de encontrar vestigios

de la Mathesis en la antigüedad, a partir de los geómetras y matemáticos. Es más, el propio filósofo francés afirma que ya había rastros de la Mathesis en pensadores como Pappus (290 d.C-350 d.C, de Alejandría, conocido por el Teorema de Pappus, y Diophanto (200/214 d.C-284/298 d.C, también de Alejandría, quien escribe su obra principal denominada Arithmética), y arguye la posibilidad de 
que sus escritos fueran escondidos debido al temor de la disminución de su valor, particularmente debido a la simplicidad y facilidad contenidas en ellas.

La Mathesis encarna la claridad del espíritu matematizado y permite el acceso a la verdad. Por lo tanto, personifica la vitalidad del proyecto cartesiano. El correlato de dicha condición para las ciencias es patente, ya que toda aquella que no disponga de la condición matematizante, no podrá llegar a conocimientos certeros. Es posible, con esto, dar cuenta de un estatus epistemológico mayor de la Mathesis por sobre otras formas de acercamiento a la res extensa. El desafío es, entonces, encauzar este espíritu, y para eso el filósofo se sirve del método, donde la certeza que entrega la Mathesis, en función de las ideas claras y distintas, la posiciona por sobre las demás ciencias, tales como la medicina, la física y la astronomía.

Descartes, a partir de la duda metódica, fundamenta la validez de las ciencias. Por ende, solo es posible, para asegurar un acervo de conocimientos válido, someter estos saberes al criterio fundamental de esta ciencia general de las cosas. A saber, "la utilidad de la duda dice relación con liberarnos de los prejuicios y nos presenta un camino muy fácil para acostumbrar nuestro espíritu a separarse de los sentidos" (Descartes 1977:17). De todo lo indicado hasta el momento, es decir, de las opiniones o de las certezas, es imposible no dudar: "En adelante, debo suspender mi juicio acerca de dichos pensamientos, y no concederles más crédito del que haría a cosas manifiestamente falsas, si es que quiero hallar algo constante y seguro en las ciencias" (Descartes 1977:20). Se traza, entonces, el acercamiento al mundo desde la duda, desde la desconfianza frente a lo percibido, y, como base del conocimiento científico. He ahí el valor de su propuesta: la duda y el cuestionamiento frente al mundo.

En la Regla II el filósofo afirma: "rechazamos todos los conocimientos tan solo probables y establecemos que no se debe dar asentamiento sino a lo perfectamente conocido y de lo que no se puede dudar" (Descartes 1996:67). Esto quiere decir que lo certero es aquello que no admite la mínima posibilidad de duda y solo no es posible dudar de aquello que se presenta con claridad y distinción. La Aritmética y la Geometría satisfacen esta condición. Para clarificar este posicionamiento, que realiza Descartes respecto de la Aritmética y la Geometría específicamente en la Regla II, es necesario dar respuesta a la interrogante de por qué estas ciencias y no otras cumplen con este principio, hecho que no deja de ser relevante en el marco de su exposición. Como mostrará el filósofo, los sentidos pueden llevar a equivocar nuestros juicios, ante lo cual afirma la posibilidad de prescindir de la experiencia como vía de acceso al conocimiento. La Aritmética y la Geometría son, en efecto, mucho más ciertas que otras disciplinas, porque solo ellas "se ocupan de un objeto de tal modo puro y simple que no suponen absolutamente nada que la experiencia haya mostrado incierto, sino que se asientan totalmente en una serie de consecuencias deducibles por razonamiento" (Descartes 1996:71).

Vale decir, la incertidumbre que puede representar la experiencia es superada por la concepción matematizante. Por ejemplo, la Geometría puede dar cuenta del largo, el ancho y el alto de las cosas, sin la necesidad de ir a ellas. De este modo, la certeza, que caracteriza a las ciencias mencionadas, se relaciona con la vía de acceso al conocimiento que estas tienen, al ocuparse de un objeto de forma pura y simple. El razonamiento deductivo, entonces, que es el utilizado por estas ciencias, entrega la garantía necesaria para el acceso a la verdad, ya que implica "todo aquello que se sigue necesariamente de otras cosas conocidas con certeza" (Descartes 1996:76). De hecho, en la deducción no es necesaria una evidencia actual, ya que deriva de un tipo de razonamiento como 
"todo aquello que sigue de otras cosas conocidas por certeza [y] es concebida como movimiento y sucesión" (Descartes 1996:76). No podría ser de otro modo, como se ha mostrado, ya que la experiencia no es requisito de acceso a la verdad para el cartesianismo, situación que se debe a la escasa confianza en los sentidos como forma de aprehensión de la realidad: "el entendimiento no es en sí mismo 'limitado', sino que opera como límite en un sujeto dotado de voluntad" (Rojas 2017:315).

Con esto, el autor no desestima la existencia de otras ciencias, vale decir, de otros objetos de conocimiento. La clave para Descartes es cómo estas ciencias (la Aritmética y la Geometría) se ocupan del objeto de estudio, aspecto que garantiza el acceso a la verdad, dando cuenta de la Mathesis Universal. En consecuencia, la naturaleza del objeto de estudio no es lo que determina la veracidad de los conocimientos, sino más bien los requerimientos que se necesitan para un correcto acceso al saber. Por lo tanto, en este aspecto es apreciable el énfasis que establece Descartes respecto de la importancia del método para el acceso a conocimientos certeros, ya que abriría la posibilidad a otras ciencias de adentrarse a la certeza matematizante, en tanto que cumpla la condición propuesta. Esto implicaría que, en el sistema cartesiano, el acceso a la verdad no lo garantiza el tipo de objeto de estudio del cual se trate, sino que el modo de acceso a estos objetos de conocimiento.

Como menciona el filósofo francés en las Meditaciones metafísicas: "la física, la astronomía, la medicina y todas las demás ciencias que dependen de la consideración de cosas compuestas, son muy dudosas e inciertas" (Descartes 1977:19). Es decir, la forma de acceso al conocimiento de estas ciencias no satisface el estado requerido, el cual es tratar de cosas claras y distintas, cuyas condiciones no permiten ningún rezago de duda. Al no cumplir con la condición mínima para el acceso a lo certero (por remitirse a cosas confusas y oscuras), estas ciencias se cierran a la posibilidad de verdad. En este aspecto, parece acertado el planteamiento de Marion, quien agrega que "lo que se juega en la Regla II no es tanto el establecimiento del paradigma matemático para todo saber, cuanto una meditación sobre la certeza como la única modalidad epistemológica admisible, sea cual sea el objeto al que se aplique" (Marion 2008:46).

A partir de la argumentación realizada, se ha ganado claridad respecto de una única vía de acceso a la verdad representada por Descartes, es decir, el acceso al conocimiento por la concepción matematizante. La Mathesis Universalis encarna la vía certera señalando en la Regla IV que "no importa si ha de buscarse en los números, en las figuras, en los astros, en los sonidos o en cualquier otro objeto" (Descartes 1996:86). El orden y la medida entregan, entonces, las garantías para un correcto camino hacia las certezas; estas condiciones implican priorizar la organización y sistematicidad en la tarea cognoscente, hecho que es relevante porque entrega validez a las ciencias, además de dar cuenta del único camino posible, estableciendo un único método común.

Es así como el conocimiento alcanzado por la Mathesis Universalis exhibe claridad sobre lo que afirma, presentando una distinción, mostrando la marca de lo simple y conduciendo a una vía de acceso certera y clara a la res extensa. En este punto, la Mathesis representa el espíritu cartesiano, y engloba un único método seguro para las ciencias. Este método, encarnado en la Mathesis cartesiana, tiene sentido en relación con la apertura al mundo exterior, de modo de reconocer un mundo existente más allá de la cosa pensante. Así, la res extensa no presupone un acceso necesario a la materialidad, ya que como se ha mostrado, los sentidos no son garantes de conocimientos ciertos. Sin embargo, Descartes realiza un tránsito hacia el reconocimiento del mundo sensible, un 
mundo particular y extraño, dando paso a la afirmación de la existencia de una extensión, unida y distinta del cogito, el que a su vez existe junto a otras extensiones. El método cobra relevancia en cuanto acceso a esa res extensa, a pesar de que la condición matematizante, como forma de conocimiento, no necesita del mundo para conocer.

Como fundamento de esta Mathesis Universalis, por lo tanto, pareciera ser que el acceso al mundo queda restringido al cogito en el sistema de pensamiento cartesiano, lo cual se refleja en el método de la duda. Que las cosas estén afuera es puesto en duda, y el mundo queda reducido al ámbito de la conciencia, sin que se tenga evidencia de las cosas que son, en cuanto no superen el ejercicio propuesto por esta ciencia general de orden superior, siempre pensando que, para Descartes, "la res cogitans, como verdad fundamental y fundamentante, posibilita la existencia de la res extensa" (Cebrecos 2014:18).

\section{El cartesianismo como pretensión de validez en la fundación de la sociología}

En la sociología científica la incidencia del cartesianismo se traduce a la necesidad de declarar, expresamente, cómo se genera conocimiento válido en una episteme referida a determinados objetos de estudio. Recogiendo parte importante de la visión cartesiana de la realidad, la sociología clásica asumió esta discusión de dos formas, cada una de las cuales representan vías distintas de acceso al conocimiento científico de los fenómenos sociales: (1) la que se realiza sobre las directrices ofrecidas a lo largo del desarrollo de la Mathesis Universalis, una tarea que fue recurrente en las primeras sociologías científicas de Comte, Spencer y Durkheim, los cuales se sintieron motivados por encontrar un objeto de estudio claro y un método de abordaje propicio para la inspección científica de estos nuevos hechos de interés; (2) la que aparece como una respuesta directa a la propuesta cartesiana del ego cogitans que muestra dificultades para acceder a la esfera de la intencionalidad, labor que en particular desarrollaron Husserl y Schütz en el seno de la fenomenología, al querer legitimar el estudio de la conciencia para abrirse no solo a una psicología trascendental, sino que a una sociología comprensiva de raíz weberiana.

En relación con la primera forma, en la sociología fundante del siglo XIX se esboza la posibilidad del abordaje científico a la realidad individual y social, manteniendo como requisito la comprobación de los hechos reales, externos al sujeto, labor que debe determinar incluso el "régimen definitivo de la razón humana" (Comte 2013a:33). Esta idea de plenitud eterna en un estadio positivo de desarrollo, postulado en los albores de la sociología, muestra claramente que la ciencia sociológica requiere de un principio cartesiano que remite a mantener una mirada racional de la sociedad, el individuo y los fenómenos sociales, "hasta que ha alcanzado las verdades más altas y más abstractas" (Spencer 1943:93).

La propuesta del positivismo es un impulso, un fuerte cuestionamiento a la metafísica, donde el esfuerzo sociológico debe estar puesto en "la subordinación constante de la imaginación a la observación, condición de toda sana especulación científica" (Comte 2013a:14). Como es lógico, toda especulación científica se logra a partir de la existencia del mundo material y de un único método generado al alero de un orden superior, donde se sustituye cualquier tipo de observación "por aquella previsión racional, que constituye, por todos aspectos, el principal carácter del espíritu positivo" (Comte 2013a:15). En una lógica evolucionista, coincide con este dictamen, toda vez que reconoce "que la tendencia a creer en la universalidad de la ley se ha hecho, de época en época, cada vez más fuerte" (Spencer 1943:103). 
Vale decir, la previsión racional subyace a la concepción de un espíritu positivo. Esto se logra a partir de método positivo, que, en la sociología de Comte, remite al principio cartesiano del orden y la sistematización de los conocimientos, instaurándose como requisito para un despliegue de este ya mencionado espíritu positivo: "el sentimiento elemental del orden es en una palabra naturalmente inseparable de todas las especulaciones positivas" (Comte 2013a:40), afirma el fundador de la sociología. Se advierten vestigios de las Mathesis Universalis, a partir de las nociones de orden y medida del pensamiento cartesiano, que son claves para dar cuenta de la ciencia positiva en Comte y Spencer, en este afán disciplinario inicial de querer alejarse cada vez más de los objetos de pensamiento metafísico, inútiles para la ciencia sociológica sustantiva. Es más, para lograr la invariabilidad de las leyes naturales, dogma del pensamiento sociológico fundacional, el sociólogo pone como requisito la exactitud frente a un orden de grandes fenómenos, y eso se ha logrado en la "fundación de la astronomía matemática durante los últimos siglos del politeísmo" (Comte 2013a:15). De esta manera, el orden, tan fundamental en el pensamiento cartesiano, se hace también necesario en el pensamiento positivo de la sociología, entendido como "un avance continuo hacia un fin determinado" (Comte 2013a:39), un proceso donde la humanidad "acabará por descubrir un orden constante de manifestaciones, hasta en los fenómenos más complejos y más oscuros" (Spencer 1943:105).

Esto quiere decir que la noción de orden, y, por lo tanto, el progreso, se relacionan inevitablemente con la pretensión de validez de la ciencia, esa que "no permitirá nunca confundir la ciencia real con esa vana erudición que acumula hechos maquinalmente sin aspirar a deducirlos unos de otros" (Comte 2013a:15). El método científico, apoyado por un razonamiento deductivo, encarna el orden necesario para la ciencia positiva en cuanto una ciencia verdadera, principio que "ha ganado poco a poco fijeza y claridad" (Spencer 1943:103). El modo de pensamiento cartesiano en esta sociología es evidente, puesto que el ya indicado orden es uno de los elementos importantes para la deducción en la Mathesis Universalis, como da cuenta Descartes en la Regla VI, al afirmar que "solo hay pocas naturalezas puras y simples que podemos intuir desde un principio y por sí mismas, independientemente de cualquier otra [y que] todas las demás, sin embargo, no pueden ser percibidas de otro modo sino que deduciéndose de estas" (Descartes 1996:91). El orden, en este aspecto, es necesario para encauzar la deducción en Descartes y lograr con éxito el despliegue del método, encontrándose en la sociología sustantiva de raíz positivista un sentido similar, en virtud de la forma en cómo se concibe la ciencia de las leyes naturales de la evolución de la sociedad.

Lo anterior responde a una necesidad urgente en el positivismo sociológico fundacional por diseñar una propuesta de explicación de las leyes naturales para dirigir el cambio social, y en este sentido, disponer de una capacidad de previsión racional de los hechos humanos, para lo cual es pertinente un razonamiento deductivo, muy intenso en esta primera sociología germinal, dedicado a "reconocer la uniformidad de las relaciones entre los fenómenos" (Spencer 1943:91). Esta tarea requiere de un acercamiento a la realidad tal, que la observación de estas leyes del movimiento de la humanidad termine siendo el gran propósito de una sociología que busca instaurarse como ciencia germinal del cambio social. De hecho, según Comte, "el método positivo ha comenzado a dirigir de modo exclusivo la actividad de nuestra inteligencia" (Comte 2013b:188).

Este tipo de afirmaciones lleva a pensar en el estatus de los conocimientos científicos. Descartes señala, en reiteradas ocasiones, que el abordaje de la res extensa prescinde de la experiencia sensible. Sin embargo, pareciera ser que la sociología empirista, si bien acepta la condición cartesiana de universalidad (de las leyes del cambio social), buscaría prescindir de esa afirmación 
cartesiana 'desdeñosa' respecto de la experiencia sensible, puesto que para los sociólogos fundadores es condición necesaria de una disciplina, que tiene pretensiones de validez científica, el establecimiento de conocimientos ciertos y claros sobre el mundo social, lo cual se asegura a través del método positivo, que jamás se separa del pensamiento: "el método y la doctrina no pueden juzgarse bien más que según sus verdaderas relaciones mutuas" (Comte 2013a:31). La relevancia del método, para el establecimiento de verdades certeras, es decir, para dictaminar conocimientos que no dejen lugar a la especulación, es parte troncal del pensamiento sociológico fundacional en su versión positivista. Esto remite a la influencia del pensamiento cartesiano, en cuanto centralidad de la Mathesis Universalis para dichos propósitos, que se asumen en la sociología como la idea de un método general de los hechos sociales.

Dado ello, conocer de forma certera remite a una concepción de la realidad objetiva que es externa al cogito, asunto que "solo puede ser concebida en los mismos términos que la relación entre los cuerpos del mundo, como relación causal" (Pavesi 2014:221). Durkheim, si bien es cierto se reconoce más deudor de Bacon que de cualquier otro pensador en el plano del método, siglos más tarde centró su objetivo en construir una epistemología y una metodología propias de la investigación sociológica, recogiendo una inquietud cartesiana esencial para el estudio de hechos sociales: "nuestro método es objetivo, está dominando completamente por la idea de que los hechos sociales son cosas y deben ser tratados como tales" (Durkheim 2001:202). Al tratar los hechos sociales como cosas, la validez científica en Durkheim deviene en objetividad, en el sentido de que la sociología científica debe encaminarse a generar conocimientos no rebatibles, ni que presupongan prenociones de quien investiga. La idea durkheimiana de validez proveniente de la generación de conocimientos objetivos es visualizada en el proyecto cartesiano. La Mathesis Universalis conduce a ideas ciertas y claras, cuya certeza es innegable al constituir verdades objetivas, en tanto que científicas. Por tanto, la pretensión de objetividad, en el pensamiento de Durkheim, se erige como una herencia cartesiana clara en materia de abordaje de los hechos sociales, en el marco de una ciencia sociológica objetiva de los hechos sociales, donde "la verdad objetiva es independiente de quien la observa, la cual puede ser cognoscible por medio de la ciencia" (Paredes y Castellanos 2011:92).

Esta constitución de la sociología científica, con Durkheim, refleja intensamente una respuesta ante esas "especies de fantasmas que nos desfiguran el verdadero aspecto de las cosas y que, sin embargo, tomamos por las cosas mismas" (Durkheim 2001:56). Para alejarse de esos fantasmas, es necesario definir y explicar de manera precisa los hechos sociales en el orden moderno, como parte de la certeza sociológica, que nos aleja de los idolas baconianos en la búsqueda de un saber científico sobre el orden social de cosas, y que retrotrae al pensamiento cartesiano y su constante reafirmación del ejercicio de la duda metódica. En Descartes, las certidumbres de los conocimientos a los que se puede llegan a partir de su superación, dan cuenta de la necesidad de poner en tela de juicio lo conocido hasta el momento, actitud científica durkheimiana hacia la realidad innegable de los hechos sociales, que instituye el alejamiento de las prenociones y de las predisposiciones subjetivas del investigador sociológico respecto de su objeto de estudio.

Resulta lógico, por ende, que el esfuerzo de Durkheim esté orientado a la realización de una ciencia que difiera de la filosofía, en cuanto al abordaje del objeto de estudio: "todo lo que pide (la sociología) es que se le conceda que el principio de causalidad se aplique a los fenómenos sociales" (Durkheim 2001:199). Estos mismos fenómenos constituyen "una nueva especie y a ellos debe darse y reservarse el calificativo de sociales" (Durkheim 2001:41). Al referirse a los hechos sociales, de ese 
modo queda en evidencia que, si bien recoger la noción de ideas certeras sobre la base de las res extensa se yergue como un postulado en la fundación de la sociología durkheimiana, se apreciaría complementariamente un alejamiento respecto de Descartes, en la medida en que, para la sociología fundacional, la naturaleza del objeto es de primera relevancia, mientras que en el pensamiento cartesiano, en cuanto fin último de conocimiento, las certezas están dadas a partir del cumplimiento de lo establecido en la Mathesis Universalis, más que en las cosas mismas. Sin embargo, ya desde Comte el volcarse al mundo objetivo es un criterio clave para consolidar la sociología, proceso que ha sido producido "mediante los progresos de la observación" (Comte 2013b:190).

En relación con la segunda forma anunciada al comienzo de esta sección, el desarrollo de la perspectiva fenomenológica se asienta en un profundo abordaje cartesiano de la esfera de la conciencia trascendental, generando importantes distancias con el enfoque propuesto por Descartes. Erigida desde una visión de la realidad psíquica y social radicalmente diferente a la de la sociología positiva, dos de sus grandes exponentes, Husserl y Schütz, reconocen las deudas intelectuales con el sistema cartesiano, más intentan una superación epistémica que tribute, sobre todo en el caso del segundo, a una sociología comprensiva intrínsecamente weberiana, que con el tiempo se ha ido posicionando como un enfoque relevante desarrollado dentro de la ciencia de la sociedad. Esto es central en la discusión sociológica, toda vez que es allí donde la disciplina "comenzó a materializar su proceso de diferenciación, sobre todo, respecto de la filosofía" (Alvear 2017:250).

A juicio de Schütz, Husserl reconoce que Descartes "no llegó a fondo en su análisis, y aunque tuvo en su mano la clave de un gran descubrimiento, vaciló en utilizarlo, en extraer sus consecuencias ineludibles" (Schütz 2003a:113). Vale decir, en esta otra modalidad de influencia del pensamiento cartesiano en sociología, es el alemán quien recoge directamente las ideas expresadas por el pensador francés, buscando sobre esa base superar su sistema filosófico, para abrirlas al mundo de la conciencia trascendental, piso indiscutible para la emergencia ulterior de una sociología fundacional de corte comprensivo. La relevancia de Descartes como punto de partida de esta propuesta de fenomenología trascendental es tal, que con Husserl se presentaría una salida a la condición matematizante como requisito de validez para todo conocimiento científico, específicamente en el sentido en que la ofrece el moravo en sus Meditaciones cartesianas. Si bien éste no renuncia a la pretensión de validez de las ciencias como fin último, rechaza enfática y sistemáticamente la vía matematizante como acceso único a las certidumbres, y con esto el axioma cartesiano como único modo para aquello, con un ego que busca "unos caminos apodícticamente ciertos por los cuales pueda franquearse en su pura interioridad su exterioridad objetiva" (Husserl 1996:39).

Para Husserl, la interioridad no es suficiente para dar cuenta del mundo cartesiano externo: "yo no puedo formular o admitir como válido ningún juicio que no haya sacado la fuente de la evidencia, de experiencias en las cuales me estén presentes respectivas cosas y hechos objetivos ellos mismos" (Husserl 1996:54). Es decir, en Husserl las cosas son dadas al acto de la conciencia, sobre la base de la operatoria de la intencionalidad, a partir de un sujeto que está abierto y vuelto hacia las cosas mismas. Esto sería lo que ignoró Descartes: "al considerar las cogitaciones que aparecen dentro del flujo de pensamiento como entidades aisladas [sin establecer tajantemente] una distinción lo bastante radical entre el acto del pensar y el flujo de pensamiento" (Schütz 2003a:114), donde la labor filosófica de Descartes, hay que recordar, siempre consistía en "el retiro del yo empírico hacia 
el hogar del fuero interno" (Rojas 2017:301). Esto no es menor para los postulados de la fenomenología trascendental y la fenomenología social de Schütz, ya de raíz propiamente sociológica, sobre todo si se piensa en que, para esta última, no solo el yo tiene esa capacidad cartesiana atribuida al cogito, sino que también los otros que habitan el mundo de la vida cotidiana: "el tú realiza también actos intencionales, confiere también significado" (Schütz 1993:128).

La sociología comprensiva weberiana, tal como en la sociología sustantiva y fundacional del positivismo durkheimiano, también sabe recoger críticamente el legado cartesiano en torno a la Mathesis Universalis, en tanto asume la condición de un ego interpretativo que reconoce una externalidad objetiva más allá del mero mundo material de los objetos naturales, con una capacidad para identificar otras esferas intencionales que, a su vez, confieren significado a su mundo subjetivo y al mundo compartido. Schütz es categórico al respecto, afirmando que "el concepto cartesiano del flujo de las cogitaciones puede ser radicalizado en gran medida señalando su carácter intencional" (Schütz 2003a:114). Esta intencionalidad, si bien estaría reconocida por Descartes, caería fuera del foco de interés del filósofo, cuando intenta demostrar que la Mathesis Universalis debe dar cuenta de un método general que se desarrolle sin atender especialmente a ningún tipo de materia en lo particular. Por el contrario, para la fenomenología social esa 'materia especial' posee una base de significado incrustada directamente en la intencionalidad como fundamento de la vida consciente de los seres humanos, a tal punto que llega a aparecer como un mundo dado previamente, sedimentado, heredado e institucionalizado, compartido con semejantes con los cuales el ego mantiene grados variados de relaciones sociales: "todos somos seres humanos en nuestra vida cotidiana, hombres entre semejantes" (Schütz 2003b:74), insistiendo en que el mundo social "está ordenado alrededor del sí-mismo como centro, en grados diversos de intimidad y anonimia" (Schütz 2003b:75). Descartes lo perfila, para luego desinteresarse: "el método cartesiano, supone un cambio artificial en la actitud que el hombre observa en la vida cotidiana" (Schütz 2003a:114).

Es decir, respecto del nivel interior del cogito, es posible afirmar que los postulados cartesianos no hacen mayor referencia a la intencionalidad como fundamento trascendental del devenir en el mundo. Para la psicología husserliana de la intencionalidad pura, no advertir dicha intencionalidad impide al filósofo abrirse significativamente a la res extensa, a esa res cartesiana que tiende a quedar desconectada del flujo puro de las cogitaciones del yo. El cogito tiende a quedarse, por decirlo así, encerrado en sí mismo, con base en el afán de ir reconociendo las certezas indesmentibles del mundo que se abre e ilumina ante la ciencia, buscando caminos para acceder, con base en la Mathesis Universalis, a saberes oscuros que deben irse iluminando gradualmente. Esto es reconocido por parte de la sociología comprensiva de Schütz, cuando afirma que es afán de la filosofía moderna de Descartes pensar que las certezas se alcanzan "dudando sistemáticamente de todas nuestras experiencias pasibles de cuestionamiento" (Schütz 2003a:113). Sin embargo, y siempre con el afán de fundamentar una sociología del mundo intersubjetivo de la vida cotidiana, el mismo autor austríaco insiste en que "el carácter intencional de todas nuestras cogitaciones supone necesariamente una distinción tajante entre el acto de pensar [...] y los objetos a los cuales esos actos se refieren" (Schütz 2003a:114).

Posicionar la tarea de la Mathesis Universalis por parte de la filosofía de Descartes como modo general de conocimiento científico (propósito para el cual debe centrarse en el proceder del cogito más que de la res extensa y sus modalidades de constitución), podría entenderse como una forma de omisión filosófica referida al papel de la intencionalidad en la instauración del mundo 
acertadamente recogida por Husserl para proseguir la línea de las meditaciones cartesianas, y que abre paradójicamente una opción para la constitución de una sociología comprensiva de fuerte raíz weberiana, que en la medida que sigue recogiendo los aportes cartesianos, alcanza un apogeo fundacional en la constitución de una sociología comprensiva weberiana y husserliana que tiene un propósito fundamental, a saber, la "observación e interpretación teórica del mundo social" (Schütz 2003b:74). Sobre esta pretensión, en definitiva, es posible una teoría de la comprensión intersubjetiva de raigambre husserliana, para la cual Schütz reconoce que debe partir del reconocimiento de un mundo social "tal como se la acepta en la actitud del punto de vista natural, sea en la vida cotidiana o en la observación sociológica" (Schütz 1993:127). Es posible, entonces, la pregunta por el cómo se constituye el mundo social intersubjetivo, y para ello, la sociología comprensiva atiende a sus pretensiones de validez recogiendo críticamente el cartesianismo.

La filosofía transcendental de Husserl, así, es el referente mediante el cual Schütz instaura su edificio conceptual, y permite reafirmar el giro del primero, respecto del pensamiento cartesiano. El sujeto trascendental cartesiano sucumbe frente al mundo de la vida del fenomenólogo, el cual se abre a la historicidad y a la cultura: "es intersubjetivo (el mundo) porque vivimos en él como hombres entre hombres, ligados a ellos por influencias y trabajo comunes" (Schütz 2003a:137). De este modo, mediado por la noción de intencionalidad, o sentido presupuesto en términos weberianos, el sujeto sale de sí mismo, del solipsismo cartesiano, y se enfrenta al mundo cotidiano de lo vivido, el cual se perfila como un universo de significación para los actores. El mundo de la vida representa el mundo intersubjetivo experimentado por el actor a partir de la actitud natural.

Si bien la actitud natural no encarna un obstáculo para el sujeto en el mundo cotidiano, sí lo es para el fenomenólogo. Frente a la actitud natural y la problemática que debe enfrentar el científico, Schütz, a propósito de Husserl, expondrá la reducción fenomenológica, modo de radicalización de la duda cartesiana, en tanto que la actitud natural implica suspender la duda de que las cosas existen. Es así como el fenomenólogo presupone "el esfuerzo radical de examinar críticamente todos los datos aparentemente dados de sus experiencias [...] y rechazar la actitud acrítica hacia el mundo" (Schütz 2003a:113). Las reverberaciones del método cartesiano son evidentes al considerar la posibilidad de certeza como fundamento de lo verdadero.

\section{Conclusiones}

La distinción cartesiana entre ideas claras y distintas permite reconocer las implicancias de su filosofía en la fundación de la sociología científica. Lo anterior, que en parte responde a un impulso comteano inicial, remite indefectiblemente a la Mathesis Universalis, como vía exclusiva a la verdad en Descartes. La validez del método, a partir de la matematización, en tanto permite satisfacer los criterios de claridad y distinción cartesianos, posibilita establecer verdades tan certeras y evidentes que sean accesibles y resistan todo tipo de cuestionamientos. De este modo, la sociología fundacional está cargada de esta herencia cartesiana, lo que supone una continuidad evidente con su pensamiento, manifestado en la noción de objetividad. En el despliegue del método científico radica un elemento clave en la sociología fundacional de matriz positivista, abrazando con ello los principios cartesianos fundamentales. Específicamente, como se ha indicado en párrafos precedentes, ya Comte y Spencer refieren insistentemente a la noción de orden y razonamiento deductivo del pensar sociológico, orientado a la enunciación de las leyes naturales del cambio social. En Durkheim, como se ha señalado, destaca la continuidad referida a la pretensión de objetividad de su sociología, insistiendo en un quiebre epistemológico con el sentido común, toda vez que 
plantea el alejamiento de las prenociones para acceder al conocimiento científico. Esto retrotrae el ejercicio la duda metódica en Descartes, de manera tal que permite la continuidad en las pretensiones de validez, que son asumidas en los albores de la sociología por sus fundadores. Por ende, la Mathesis Universalis estaría situada en la raíz constitutiva misma de estas relevantes áreas de conocimiento sociológico.

No obstante, estarían presentes también algunas discontinuidades o divergencias en relación a la misma Mathesis Universalis, hecho que estaría asociado sobre todo a la intención de reconocer un orden muy diverso de objetos susceptibles de ser aprehendidos por diferentes ciencias. El análisis realizado permite distinguir la necesidad de un campo de estudio propio de la naciente disciplina, que desea entenderse y proyectarse como una ciencia de lo social. Según se ha planteado, la Mathesis Universalis delimita un único modo epistemológico plausible de acceder a la res extensa, sea cual sea el objeto al que se aplique. La sociología, en tanto, delimita con urgencia un claro y único objeto de estudio: las leyes universales del cambio social, por parte de Comte y Spencer, y los hechos sociales, por parte de Durkheim. Esto no es menor, ya que hay un esfuerzo constante en estos pensadores por proporcionar un campo de estudio propio para una naciente disciplina, exclusivo respecto de otros órdenes de fenómenos y que queda disponible para una naciente ciencia de la sociedad. Por lo tanto, tomando distancia del planteamiento cartesiano, el objeto sí importa, y esta relevancia fundacional se adhiere a la intención de los teóricos del positivismo fundacional que es, precisamente, definir un ámbito exclusivo de lo social, es decir, un objeto de estudio propio. Esta labor ha generado la exclusión inmediata de otro tipo de objetos de estudio del campo de interés sociológico, debido a que no tienen un origen netamente social.

Cabe afirmar una continuidad crítica notoria del pensamiento cartesiano, tanto en la fenomenología trascendental husserliana como la fenomenología social schütziana. Esta continuidad crítica sería, incluso, más explícita en estos edificios epistémicos que en el positivismo sociológico de consolidación durkheimiana, bajo el modo de una psicología trascendental husserliana y una sociología comprensiva edificada por Schütz sobre la base del pensamiento weberiano, que se esfuerza consiente e insistentemente por recoger la esfera de la intencionalidad como campo determinante del análisis fenomenológico, lo cual se aplicaría tanto para el ego cogitans como la res extensa, ambas inundadas por dicha intencionalidad. La trascendencia que ha tenido el desarrollo ulterior de la fenomenología social en el campo de la sociología comprensiva (entendida, incluso, como una 'sociología cualitativa' o 'sociología interpretativa'), esto es, en una praxis investigativa de fuerte raíz empírica y casuística, obliga a pensar recurrentemente en el impacto que el pensamiento cartesiano ha tenido para el ejercicio mismo de la disciplina. Tal como ocurre con el positivismo durkheimiano, si bien la naturaleza misma de esta relevante línea de pensamiento sociológico no le debe solo a Descartes el erigirse sobre la base de fundamentos epistemológicos sólidos (puesto que ambos enfoques reciben múltiples influencias), cabe encontrar en los postulados del francés un espacio conceptual y metodológico lúcido, vanguardista y útil que tiene una capacidad notable para traspasar las barreras del tiempo.

\section{Agradecimientos}

Beca Doctorado, Agencia Nacional de Investigación y Desarrollo de Chile (ANID-PFCHA/Doctorado Nacional/2018-21180055). 


\section{Bibliografía}

Alvear, R. 2017. La sociología clásica y el destierro del ser humano. Cinta de Moebio 59: 235-253. https://doi.org/10.4067/S0717-554X2017000200235

Cebrecos, F. (2014) Descartes: entre la psicología racional y la antropología filosófica. Persona 17: 11-32. https://doi.org/10.26439/persona2014.n017.286

Comte, A. (2013a) Discurso sobre el espíritu positivo. Globus.

Comte, A. (2013b) Primeros ensayos. Fondo de Cultura Económica.

Descartes, R. (1977) Meditaciones metafísicas con objeciones y respuestas. Ediciones Alfaguara.

Descartes, R. (1996) Reglas para la dirección del espíritu. Alianza Editorial.

Durkheim, E. (2001) Las reglas del método sociológico. Fondo de Cultura Económica.

González, R. (2016) Dos criterios para la presencia de estados mentales: Descartes y Turing. Cinta de Moebio 56: 159-171. https://doi.org/10.4067/S0717-554X2016000200004

Hegel, G. (1984) Introducción a la historia de la filosofía. Guillermo Escolar.

Husserl, E. (1996) Meditaciones cartesianas. Fondo de Cultura Económica.

Marion, J. (2008) Sobre la ontología gris de Descartes. Ciencia cartesiana y saber aristotélico en las Regulae. Escolar y Mayo Editores.

Paredes, G; Castellanos, C. (2011) Principios filosóficos del pensamiento positivista de Auguste Comte. Academia 10(19): 90-101. http://erevistas.saber.ula.ve/index.php/academia/article/view/6088

Pavesi, P. (2014) Cuerpo y carne en Descartes. Ideas y valores 63(155): 219-234. https://doi.org/10.15446/ideasyvalores.v63n155.42528

Rojas, S. (2017) La cosa que piensa: una lectura de las Meditaciones Metafísicas de Descartes. Revista de Filosofía 73: 299-316. https://doi.org/10.4067/s0718-43602017000100299

Schütz, A. 1993. La construcción significativa del mundo social. Introducción a la sociología comprensiva. Paidós.

Schütz, A. (2003a) El problema de la realidad social. Escritos I. Amorrortu Editores.

Schütz, A. (2003b) Estudios sobre teoría social. Escritos II. Amorrortu Editores.

Spencer, H. (1943) Clasificación de las ciencias. Anaconda.

Recibido el 16 Jun 2020

Aceptado el 23 Oct 2020 